\title{
A Multi-spectral Test and Simulation Facility to Support Missile Development, Production, and Surveillance Programs
}

\author{
James B. Johnson, Jr. ${ }^{1}$ and Jerry A. Ray ${ }^{2}$ \\ ${ }^{1}$ U.S. Army Developmental Test Command, Redstone Technical Test Center, \\ CSTE-DTC-RT-E-SA, Redstone Arsenal, AL 35898, USA \\ James.Johnson@rttc.army.mil \\ ${ }^{2}$ U.S. Army Aviation and Missile Command, Research, Development and \\ Engineering Center, AMSAM-RD-SS-HW, Redstone Arsenal, AL 35898, USA \\ jerry.a.ray@redstone.army.mil
}

\begin{abstract}
The Multi-Spectral Missile Life-Cycle Test Facility (MSMLCTF) is a single hardware-in-the-loop (HWIL) simulation complex that will uniquely have the capability to perform life-cycle simulation/testing on next-generation multi-sensor missile systems. The facility will allow for simulated end-to-end flight tests in a non-destructive manner as well as complete evaluation of all missile electronics and software. This testing will be performed at both the AllUp-Round (AUR) level and the Guidance Section (GS) level. The MSMLCTF will be used to support the Army Transformation by evaluating operational flexibility before fielding, reducing life-cycle costs, improving system performance by evaluating technology insertions and evaluating interoperability impacts. This paper gives a general description of the MSMLCTF, cost tradeoffs between live flight testing and simulation, and an update on facility development status.
\end{abstract}

\section{Introduction}

In the past, missile systems were tested solely at an open-air flight range. This technique was relatively simple. In recent years, missile systems have become much more complex utilizing multiple sensors such as millimeter wave and infrared. Also in the past, weapon developers developed and used simulation facilities to develop a weapon and testers developed and used simulation facilities to test a weapon. This paper describes a new multi-spectral simulation facility which will be utilized to both develop and test next-generation multi-spectral missile systems.

\section{Historical Perspective}

The authors played an integral role in developing two previous simulation facilities the Millimeter System Simulator One (MSS-1) and the Simulation/Test Acceptance

V.N. Alexandrov et al. (Eds.): ICCS 2001, LNCS 2074, pp. 515-520, 2001.

(C) Springer-Verlag Berlin Heidelberg 2001 
Facility (STAF). Lessons were learned in the development of these facilities which led us to the development of the Multi-Spectral facility. A brief description of each of these facilities is provided.

\subsection{Millimeter System Simulator One (MSS-1)}

The MSS-1 facility was developed by the U.S. Army Aviation and Missile Command's (AMCOM) Research, Development and Engineering Center (RDEC) in the mid-1980's primarily for the Longbow HELLFIRE missile system guidance electronics (no explosives). The Longbow HELLFIRE is a millimeter wave radar guided system. This facility featured a high fidelity target array, flight motion simulation, and signal injection. The principal utility of the facility was development of the weapon system in a repeatable edge-of-the-envelope environment. Capabilities included end-to-end hardware-in-the-loop simulations using multiple targets and countermeasures. Early prototype guidance systems were placed in the facility for development of weapon system software algorithms. Also, many guidance packages scheduled to be flown on a missile system were placed in the facility to judge flight readiness. The missile system is in production today; however, guidance packages are still inserted into the facility to periodically check edge-of-the-envelope performance. An artist's sketch of the facility is shown in Figure 1.

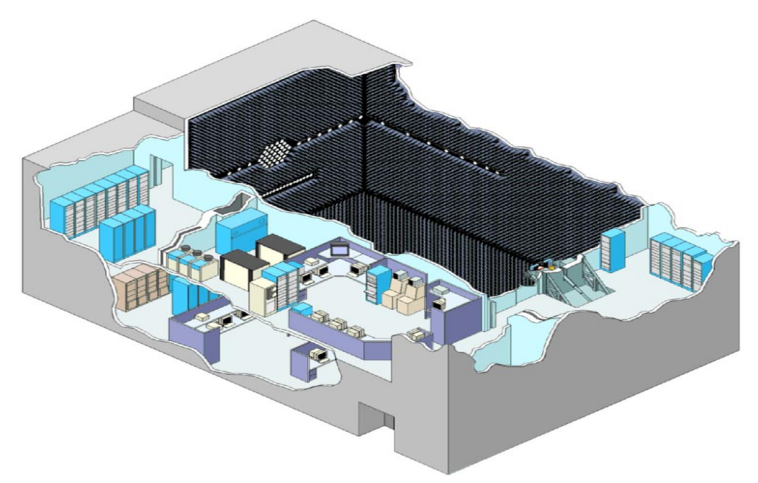

Fig. 1. Millimeter-wave System Simulator One (MSS-1) Facility

\subsection{Simulation/Test Acceptance Facility (STAF)}

The STAF facility was developed jointly by the U.S. Army Developmental Test Command's (DTC) Redstone Technical Test Center (RTTC) and AMCOM RDEC in the mid-1990's. The mission of STAF was to perform functional testing on Longbow 
HELLFIRE missiles (complete all-up-rounds with explosives). The STAF operated in a similar fashion to the MSS-1 facility with the exceptions that the missile was complete with explosives installed and the target array was much more simplistic. The target array was deemed adequate for a production environment. The facility, still in use today, is used for production sampling, special engineering tests, and stockpile surveillance testing. An artist's sketch of this facility is shown in Figure 2.

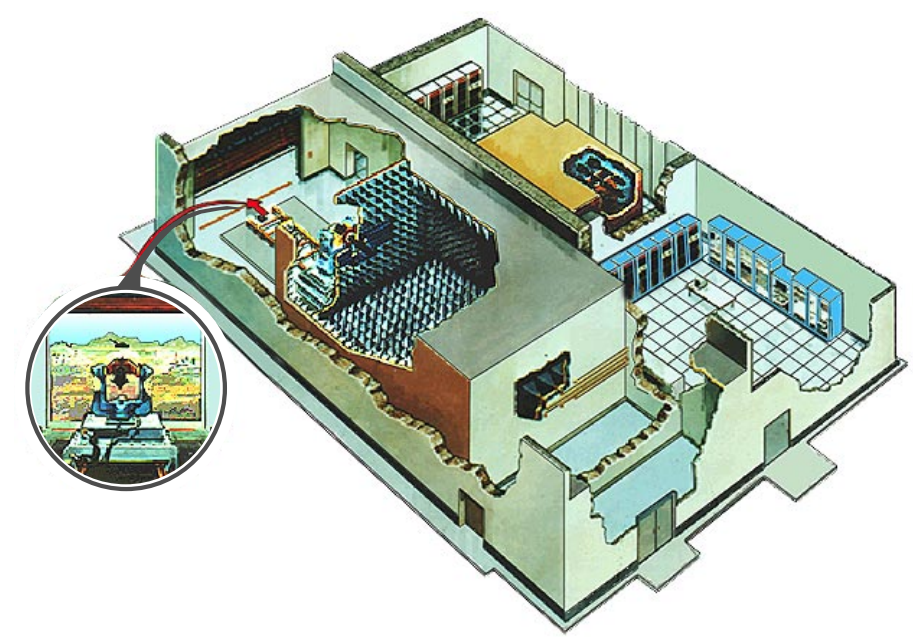

Fig. 2. Simulation/Test Acceptance Facility (STAF)

\section{Next Generation Multi-spectral Simulation Facility}

Many lessons were learned in the development of the MSS-1 and STAF facilities. Emerging weapon system technologies indicate the requirement for a Multi-Spectral hardware-in-the-loop simulation facility. Multi-Spectral implies the incorporation of both Millimeter Wave and Imaging Infrared in the same guidance package. As such, the simulation facility must be capable of providing multi-spectral targets.

\subsection{Concept}

Taking advantage of the lessons learned on the MSS- 1 and STAF facilities, the authors devised a concept for the Multi-Spectral facility which houses both a nonhazardous high-fidelity facility and a hazardous lower-fidelity facility. The facility will consist of two test chambers, one high fidelity and the other lower fidelity, with a common control building. The high fidelity chamber will be used primarily for missile development and will feature multi-spectral multiple targets, countermeasures, and edge-of-the-envelope evaluations. This facility will be used to design hardware 
and develop software algorithms. The lower-fidelity chamber will be used for preflight confidence testing, production lot sampling, stockpile reliability tests, and special engineering tests. The multi-spectral targets in this facility will be more simplistic relative to the high fidelity chamber.

\subsection{Facility Description}

A simplified block diagram of the facility is shown in Figure 3. The general concept of operation is similar to the MSS-1 and STAF facilities. The missile under test is provided with every stimuli it is capable of sensing. The missile is placed on a threeaxis flight motion simulator (pitch, yaw, and roll). The sensor of the missile is inside an anechoic chamber. An exotic material is placed inside the chamber between the sensor and millimeter wave target generation horns. The exotic material acts as a window to millimeter wave energy and a mirror to infrared energy. A dynamic infrared scene projector (DIRSP) is placed off-axis and aimed at the exotic material. Inertial navigation information, driven by a six-degree-of-freedom, is injected into the missile to simulate flight. Proper millimeter wave, infrared, and laser targeting is supplied by the respective target generators. Fin commands are extracted from the missile and this information is computed in an airframe model which in turn supplies fin position information. This information is used to drive the three-axis flight motion simulator. All of these activities occur from simulated trigger pull to simulated target impact.

\subsection{Facility Utilization}

Multiple weapon systems are being targeted for test in the Multi-Spectral facility. During system development and demonstration phases, the facility will be used to aid in system design (hardware and software) and integration for system breadboards. During the final stages of this acquisition phase, the facility will be used for pre-flight risk reduction testing for all guidance sensors and all-up-rounds prior to live flight testing. The facility will be made available to operational testers to augment their operational test scenarios and possibly reduce operational flight test requirements. The facility will be used for lot acceptance testing, engineering evaluations/tests, and stockpile reliability tests during the Production and Deployment phases. During all phases, the facility will be used to evaluate operational flexibility before fielding, reducing life-cycle costs, improve system performance by evaluating technology insertions, and evaluating interoperability impacts. 


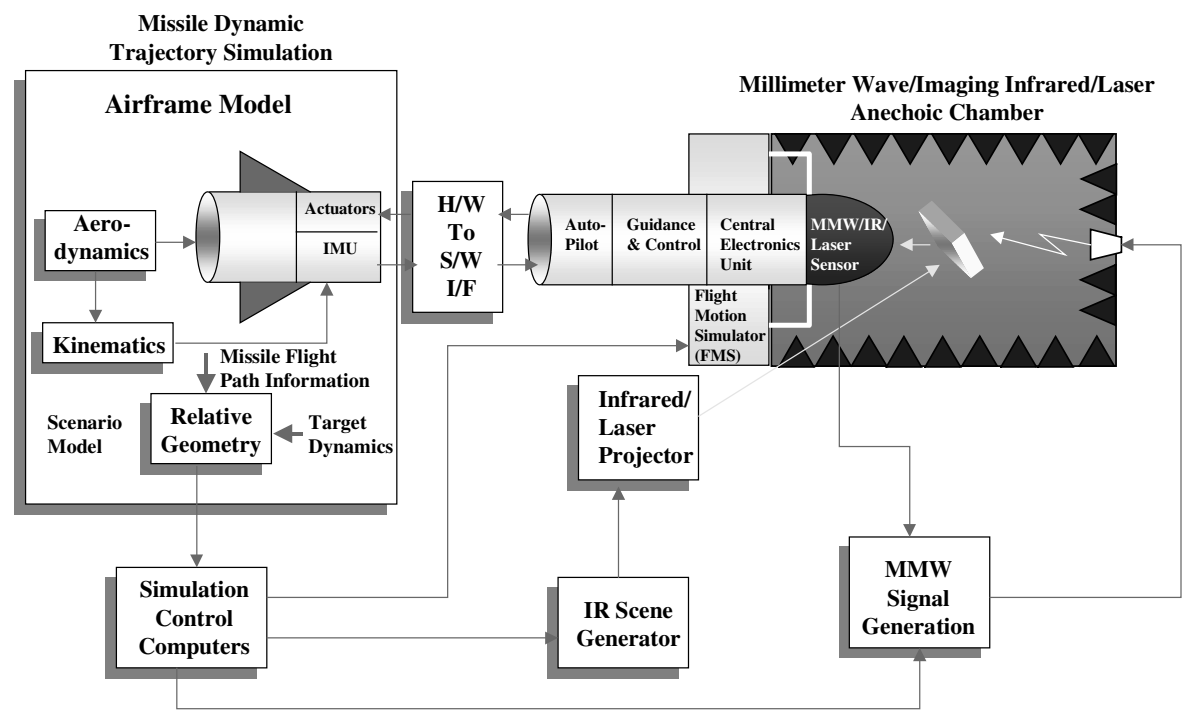

Fig. 3. Multi-Spectral Simulation Facility Block Diagram

\subsection{Cost Avoidance}

The authors performed a cost tradeoff of performing only a flight test program at a test range versus performing a combination of flight testing and hardware-in-the-loop simulation testing.

Some assumptions that went into this analysis for each phase of the weapon life cycle were: (1) the number of missiles that would be fired per year with no hardware-inthe-loop simulation, (2) the number of missiles undergoing hardware-in-the-loop simulation per year in the multi-spectral simulation facility as well as the number of missiles actually fired as augmentation. The results of the cost tradeoff using conservative values result in a cost avoidance of at least $\$ 90 \mathrm{M}$ over the life of a weapon system with potential of up to $\$ 100 \mathrm{M}$. The tremendous cost avoidance is due to the non-destructive nature of the simulated flights which allows the rounds to be placed in inventory upon test completion. This same analysis indicates a facility cost payback period of less than five years.

\section{Conclusion}

This paper gave a historical perspective on the development of hardware-in-the-loop simulation facilities to test missile systems. Lessons learned have been applied to a 
new facility, under development, to test multi-spectral missile systems. An overall description of how this facility works, how it will be utilized, and the benefits to the U.S. Army have been provided. 